\title{
1 Flies improve the salience of iridescent sexual signals by orienting
}

\section{2 toward the sun}

3

4 Thomas E. White ${ }^{1,2}$, Tanya Latty ${ }^{1}$

$5 \quad{ }^{1}$ School of Life and Environmental Sciences, The University of Sydney, Sydney, Australia 2106

$6 \quad{ }^{2}$ Corresponding author.

8 E-mail: thomas.white@sydney.edu.au

9 Keywords: sexual selection, structural colouration, wing interference pattern, sensory drive

10 Word count: 4516

11 Number of figures: 4

12 Number of tables: 1

13 Supplementary information: supplementary figure \& table 


\section{Abstract}

19 Sunlight is the ultimate source of most visual signals. Theory predicts strong selection for its

20 effective use during communication, with functional links between signal designs and display

21 behaviours a likely result. This is particularly true for iridescent structural colours, whose

22 moment-to-moment appearance bears a heightened sensitivity to the position of signallers,

23 receivers, and the sun. Here we experimentally tested this prediction using Lispe cana, a muscid

24 fly in which males present their structurally coloured faces and wings to females during ground-

25 based sexual displays. In field-based assays we found that males actively bias the orientation of

26 their displays toward the solar azimuth under conditions of full sunlight and do so across the

27 entire day. This bias breaks down, however, when the sun is naturally concealed by heavy cloud

28 or experimentally obscured. Our modelling of the appearance of male signals revealed clear

29 benefits for the salience of male ornaments, with a roughly four-fold increase in subjective

30 luminance achievable through accurate display orientation. These findings offer fine-scale,

31 causal evidence for the active control of sexual displays to enhance the appearance of iridescent

32 signals. More broadly, they speak to predicted coevolution between dynamic signal designs and

33 presentation behaviours, and support arguments for a richer appreciation of the fluidity of visual

34 communication. 


\section{Introduction}

39 The structure of any effective communication system is shaped its response to basic

40 challenges. Encoding information (Weaver et al. 2018; White 2020), ensuring its delivery

41 (Endler and Thery 1996), and separating signal from noise (Warrant 2016) are essential features

42 of signalling systems, and selection has generated diverse solutions. In general terms, theory

43 predicts the coevolution of signals and signalling behaviours that secure the exchange of

44 information between interested parties while minimising exposure to eavesdroppers (Endler

45 1992; Endler 1993). Males of the common eggfly Hypolimnas bolina, for example, combine

46 limited-view iridescent colours with precision display behaviours to deliver maximally

47 conspicuous signals during courtship (White et al. 2015). Great bowerbirds, by contrast, present

48 coloured objects to females from within constructed viewing 'theatres', and in doing so control

49 both the object of display and the broader visual environment (Endler et al. 2010; Kelley and

50 Endler 2012).

51 Sunlight is the ultimate source of most visual signals, as its colour and intensity shape the

52 appearance of reflective surfaces. Selection for its effective use should therefore be intense

53 (Endler 1992; 1993). This is particularly true for signal designs whose moment-to-moment

54 appearance bears a heightened sensitivity to local conditions, such as structural colours

55 (Kinoshita 2008; Mouchet and Vukusic 2018). Structural colours result from the selective

56 reflectance of light by tissues organised at the nanoscale and can achieve levels of brightness and

57 chromaticity that are unattainable with pigments alone (Kinoshita 2008; Johnsen 2012). Where

58 coherent or quasi-coherent scattering is involved, as is most often the case, they are also capable

59 of iridescence and limited-view expression (Prum 2006; Vukusic 2006). 
Given that the optimal colour and/or brightness of iridescent signals is tied to the

61 geometry of the signaller, viewer, and illuminant, its widespread use in nature suggests the

62 existence of flexible behaviours for their effective presentation. That is, signallers should seek to

63 capture available sunlight so as to maximise the conspicuousness of structurally coloured signals

64 to viewers (Endler 1992; Endler and Thery 1996). This prediction has found broad support

65 across model vertebrates with observational evidence showing how signallers vary the timing,

66 location, or orientation of their displays to improve communication efficacy (Endler 1991;

67 Endler and Thery 1996; Dakin and Montgomerie 2009; Bortolotti et al. 2011; Sicsu et al. 2013;

68 Klomp et al. 2017; Simpson and McGraw 2018; O’Neill et al. 2019). Though this work is

69 extremely valuable, a richer causal understanding awaits manipulative tests across temporal and

70 taxonomic contexts.

71 Flies rank among the most diverse animal orders and this is reflected in the traits that

72 have evolved in the service of visual communication (Marshall 2012). Sexually- and

73 ecologically- specialised eye structures (Zeil 1983; Hardie 1986), elaborate colour patterns

74 (Shevtsova et al. 2011; Butterworth et al. 2020), and ritualised display behaviours (Land 1993;

75 Zimmer et al. 2003; Lunau et al. 2006; Butterworth et al. 2019) abound, and offer tractable but

76 underutilised opportunities for general tests of theory. Work on visual signalling in the group

77 continues to document the widespread presence of conspicuous structural colours, with recent

78 efforts highlighting 'wing interference patterns' (WIPs) as potential vectors of information

79 (Shevtsova et al. 2011; Katayama et al. 2014; Hawkes et al. 2019; Butterworth et al. 2020).

80 These striking patterns are the result of thin film interference from chitin/air interfaces in the

81 wing membrane (Shevtsova et al. 2011) and they are regularly presented during courtship (Land

82 1993; Frantsevich and Gorb 2006; Katayama et al. 2014; White et al. 2020). The wings bearing 
83 them are also typically transparent and transmit ca. $70-80 \%$ of incident light. This combination

84 of iridescence and transparency makes their appearance uniquely environmentally contingent

85 since bright visual backgrounds or the misalignment of signallers, receivers, and the sun will

86 render the patterns invisible. The significance of visual backgrounds during signalling is well

87 demonstrated (Katayama et al. 2014) and includes evidence for active choice in the wild (White

88 et al. 2020). Whether and how signallers actively use the sun to improve communication,

89 however, is unknown.

90 The genus Lispe is a cursorial group of muscid flies comprised of at least 163 species

91 spanning most terrestrial biogeographic regions (Pont 2019). The group showcases striking

92 interspecific and sexual variation in colouration and displays (Frantsevich and Gorb 2006; Pont

93 2019). Lispe cana is an exemplar endemic to the foreshores of Australia's east coast. Males and

94 females of the species bear structurally coloured UV-white and yellow faces, respectively, as

95 well as dimorphic wing interference patterns (unpublished data). During their ground-based

96 courtship male Lispe cana approach and straddle females from behind and maintain this position

97 as females walk about the environment. They then release and move directly in front of females

98 to present their faces and wings in a ritualised 'dance' (White et al. 2020). Males can and do

99 enhance the salience of their signals through the selection of backgrounds against which to

100 display, and similar potential benefits exist through the careful use of sunlight.

101 Here we use observational and manipulative field-based assays at a fine temporal scale to

102 test the hypothesis that males should orient their displays toward the sun so to improve the

103 salience (and, hence, attractiveness) of their iridescent signals. This predicts a bias in the

104 orientation of male displays toward the sun's azimuth position under clear skies, when the its

105 position is readily visible and the potential benefits for signal salience are likely maximised. As a 
106 corollary, the natural or artificial removal of direct sunlight should disrupt any orientation bias

107 by either impeding male's ability to orient and/or diminishing the benefits of such behaviour for

108 the salience of signals.

\section{Methods}

\section{Sexual displays and orientations}

111 Courtship in Lispe cana proceeds in four general stages: (1) males detect females when

112 both parties are walking about their foreshore habitats; (2) males approach females from behind,

113 before 'straddling' them and holding their wings closed as they continue to walk about the

114 habitat; (3) males release females and rapidly move immediately in front of them at close

115 distance $(<5 \mathrm{~mm})$, where they present their colourful wings and faces; (4) either the sequence

116 returns to (2) with male re-straddling females, they mate, or (most often) they disperse. The

117 second stage — straddling — thus offers males the opportunity to assess the environment and their

118 orientation within it, while the third stage is when their decision to display at a given location

119 and orientation is realised and is therefore the focus of our observations as detailed below.

120 We observed courtships in a population of L. cana that inhabits the supralittoral zone of

121 Toowoon Bay, NSW, Australia (33.36 S, 151.50 E), in September and October of 2019. During

122 each courtship, we recorded the orientation of the male during the display phase (described

123 above; Fig. 1) of their ritualized dance, which we observed at a distance of 1-2 m. Courting pairs

124 were haphazardly selected during slow, repeated walks by an observer along a $255 \mathrm{~m}$ transect at

125 a distance of 5-10 $\mathrm{m}$ from the shoreline. Once a courtship naturally terminated with both parties

126 dispersing, we placed a straight marker at the males' final position in alignment with their medial

127 body axis, before recording the markers' orientation to the nearest one degree using a digital 
128 compass, as well as the time. To initially validate the accuracy of this approach we tested it

129 against a video-based method in which male orientations were extracted from still-frames taken

130 from 25 video-recorded courtships (with a GoPro Hero 6 at 30fps) in which a compass was

131 visible in-frame. We found a strong correlation between orientations collected from video versus

132 direct observation (Pearson's $\mathrm{r}=0.88$ ), so we favoured the latter method for its convenience in

133 garnering a large sample size.

Once courtships were observed, we used the time and location data to calculate the solar

135 azimuth and elevation during every courtship via the package 'suncalc' (v. 0.5.0; Thieurmel and

136 Elmarhraoui 2019) for R (v. 3.6.0; R Core Team 2018). We then converted our circular measure

137 of male display orientations to a linear measure of the absolute deviation of each display from

138 the sun's azimuth. This gives a value between $0-180^{\circ}$, with $0^{\circ}$ being a male displaying exactly

139 toward sun, and $180^{\circ}$ directly away from it. We ignore the position of females since they remain

140 approximately opposite males at $<5 \mathrm{~mm}$ distance during displays (Fig 1).

141 To test the extent to which males can and do orient their displays we used the general

142 procedure above across three conditions, both observational and manipulative. In the two

143 observational conditions we recorded displays between the hours of 0730-1630 either on days of

144 full direct sunlight $(n=451$ observations across six days), or heavy cloud with the sun

145 completely obscured $(n=227$ across three days). For the manipulative assay we repeated the

146 procedure as for full sun conditions but obscured any direct sunlight using a static black plastic

147 sheet (Monarch pty. 1td) suspended on four aluminium poles ca. $10 \mathrm{~m}$ from the shoreline. This

148 cast a ca. $3 \times 3$ m shadow throughout a given day, and we recorded only courtships that began

149 and finished within this shaded area $(n=191$ across three days). The broader environmental 
150 conditions were similar across days within treatments (summarised in supplementary table S1)

151 and is partially accounted for in our statistical analyses (below).

\section{Solar orientation and signal luminance}

To model the effects of male orientation on the salience of their signals we first recorded

155 vector irradiances at the study site in $10^{\circ}$ increments from $0^{\circ}$ (directly toward the sun) to $180^{\circ}$

156 (directly away) at 0800, under representative conditions of full sunlight and heavy cloud. We

157 collected all spectra using an OceanInsight JAZ spectrophotometer with cosine-corrector and

158 oriented the $180^{\circ}$ collector perpendicular to the ground (i.e. in the same plane as male faces and

159 wings during display). We then recorded the facial and whole-wing reflectance of 50 male $L$.

160 cana using the JAZ with its pulsed-xenon light source, at an integration time of $40 \mathrm{~ms}$ and a

161 boxcar width of 2 . We used a bifurcated probe to both deliver and collect light, which we

162 oriented normal to the face and wing planes at a working distances of ca. $5 \mathrm{~mm}$. These spectra

163 were lightly LOESS smoothed before being averaged to generate a representative male facial and

164 whole-wing reflectance spectrum (Fig. S1).

165 We then estimated how the luminance of male wings and faces varies as a function of

166 male orientation toward the sun. Here and throughout we focus on luminance alone given its

167 importance over chromaticity (hue and saturation) for mating (White et al. 2020). To do so we

168 calculated the integrated product of solar irradiance at each $10^{\circ}$ increment from the solar

169 azimuth, male facial or wing reflectance, and the Rh1-6 'achromatic' receptor absorbance of

170 Musca domestica (as the nearest analogue to Lispe). We conducted all spectral processing and 
171 visual modelling using lightr v1.1 (Gruson et al. 2019b) and pavo v2.4.0 (Maia et al. 2019) for R

172 v3.6.1 (R Core Team 2018).

173

\section{Statistical analyses}

175 To estimate whether male Lispe cana bias the orientation of their sexual displays toward

176 the sun we used a generalized linear mixed-effect model fit by restricted maximum-likelihood,

177 with Gaussian error and an identity link function. We specified male deviation from the solar

178 azimuth (0-180) as the response which we square-root transformed. We did so after our

179 inspection of errors from an untransformed model suggested slightly non-normal and

180 heteroscedastic residuals, which were improved to within acceptable margins (as visually

181 assessed) following transformation.

182 We specified the interaction between experimental condition (sun, cloud, and sun-

183 blocked) and solar elevation as a fixed effect, along with the main effects of each. If males do

184 actively orient their displays the result should be a negative effect of the full sunlight condition

185 on male display offset (i.e. a tendency toward smaller offsets from the solar azimuth in full sun),

186 when compared to the cloud and sun-blocked conditions. Our inclusion of solar elevation tests

187 for reduced display accuracy at higher solar elevations when the sun's azimuth is less readily

188 discerned. Such an effect would only manifest under conditions of full sunlight when any

189 display-orienting takes place, however, hence its inclusion via an interaction term. We specified

190 the Julian date on which each measurement was taken as a random effect to account for variation

191 associated with unmeasured within-day variables, such as broader climatic conditions. 
To estimate the effects of male display orientation on signal luminance we first ran

193 generalised additive models (GAM) fit by restricted maximum-likelihood, with the offset from

194 solar azimuth (in $10^{\circ}$ increments) as the lone predictor of signal luminance. The four resulting

195 models describe the possible variation in male facial and wing luminance as a function of their

196 sexual display orientation, during conditions of full sun and heavy cloud. We then used the

197 results of the full-sun GAM alone to predict the luminance of male signals at their actual

198 recorded orientations in both full-sun and heavy cloud conditions. We used only the full-sun

199 GAM after finding no relationship between signal luminance and orientation under heavy cloud

200 (Fig. 3d), and thus no possible benefits for signal salience to orienting toward the sun in such

201 conditions (see results). We then used Kruskal-Wallis rank sum tests, with eta ${ }^{2}$ as an effect size,

202 to estimate the difference in achieved luminance between male displaying under full sun and

203 heavy cloud, with their luminance predicted from the full sun GAM. This, in effect, answers the

204 question of what males achieve by orienting their displays under ideal conditions.

206 Data availability

207 All raw data and code are available via GitHub (https://github.com/EaSElab-18/ms_sunnyflies) 208 and will be persistently archived upon acceptance.

$210 \quad$ Results

211 We found clear differences in the orientation of male sexual displays across conditions

212 (Fig. 1; Fig. 2). A likelihood-ratio test on the overall model identified a significant effect of 
213 experimental treatment alone $\left(\chi^{2}=86.86\right.$, $\left.\mathrm{df}=2, p<0.001\right)$, with no evidence for an effect of

214 solar elevation $\left(\chi^{2}=2.54, \mathrm{df}=1, p=0.111\right)$ nor any interaction between treatment and solar

215 elevation (Fig. $3 ; \chi^{2}=0.81$, df $=2, p=0.665$ ). Individual estimates (Table 1) show this was

216 chiefly driven by a bias toward smaller angular offsets by displaying males under full-sun

217 conditions alone ( $\mathrm{est}=-3.11 \pm 1.136, \mathrm{df}=9, \mathrm{t}=-2.74, p=0.02$ ). Male displays were oriented

218 toward the sun at a median offset of $44^{\circ} \pm 38$ s.d. on days in which it was fully visible. But this

219 effect was absent in conditions of heavy cloud $\left(87^{\circ} \pm 50\right.$ s.d.) and artificially obstructed sunlight

$220\left(96^{\circ} \pm 52\right.$ s.d. $)$, which did not statistically differ from one another.

Our GAMs showed that the luminance of male wings $\left(\mathrm{F}_{7.94}=356, \mathrm{p}<0.001\right)$ and faces

$222\left(\mathrm{~F}_{7.94}=355.50 \mathrm{p}<0.001\right)$ follow a near-identical sigmoidal relationship with solar orientation

223 when illuminated in full sunlight (Fig. 4c). They are maximally luminant at $0^{\circ}$ from the solar

224 azimuth, inflect at ca. $45^{\circ}\left(95 \% \mathrm{CI}=37-53^{\circ}\right.$ faces, $43-47^{\circ}$ wings $)$, before reaching their minima

225 at ca. $80^{\circ}$. The luminance of wings, as compared to faces, varied across a slightly compressed

226 range owing to their reduced reflectance (supplementary Fig. S1), but was otherwise strongly

227 correlated with facial luminance. This effect was entirely absent in conditions of heavy cloud

228 (Fig. 4d), as we found no relationship between either of facial $\left(F_{1}=0.190, p=0.669\right)$ or wing $\left(F_{1}\right.$

$229=0.192, \mathrm{p}=0.667)$ luminance and solar orientation.

230 Our subsequent test of signal luminance during actual male displays suggest clear

231 benefits to active solar orientation in full sun. Both their faces $\left(\chi^{2}=86.97, \mathrm{df}=1, \mathrm{p}<0.01, \eta^{2}=\right.$

$2320.127)$ and wings $\left(\chi^{2}=86.70, \mathrm{df}=1, \mathrm{p}<0.01, \eta^{2}=0.126\right)$ were subjectively brighter, with an

233 approximately four-fold increase as a result of their active display orienting. Given the absence

234 of a relationship between signal luminance and solar orientation under heavy cloud (as above),

235 any active orientation by males could yield no benefits to signal salience in such conditions. 


\section{Discussion}

The intensity and composition of sunlight shapes the salience of visual signals, and

239 selection should favour its flexible use. Here we tested this prediction in the cursorial fly Lispe

240 cana whose structurally coloured faces and wings are central to their ritualised courtship

241 displays. Our field-based observations show that males bias the orientation of their signals

242 toward the sun under clear skies, with individuals displaying at approximately $44^{\circ}$ offset from

243 the solar azimuth on average. This degree of precision is maintained throughout the day

244 irrespective of solar elevation. Notably, however, the directional bias in displays broke down in

245 conditions of heavy cloud, and we were able to reconstruct this effect by experimentally

246 obstructing the sun under otherwise-ideal conditions. Our visual modelling of signal luminance

247 identified clear benefits to male display behaviour, with an approximately four-fold difference in

248 the fly-subjective luminance of male signals across the $180^{\circ}$ range of possible orientations. These

249 combined results offer causal evidence that male flies actively orient their conspicuous displays

250 toward the sun, with tangible consequences for the salience of their structurally coloured signals.

251 Below we consider our findings in turn and discuss their relevance for communication efficacy

252 and signal evolution more broadly.

253 One immediate question is how male L. cana reliably orient their displays throughout the

254 entire day. The sun is the brightest spot in the sky so tracking its position directly is an obvious

255 solution, and this information can be supplemented through the use of indirect cues such as

256 intensity, chromaticity, and polarization gradients. On clear days, the intensity of sunlight

257 increases predictably toward the solar azimuth and atmospheric scattering means the sky is 
258 relatively richer in long-wavelength light in the solar, as opposed to anti-solar, hemisphere (Fig.

259 3a; Coemans et al. 1994). The degree of polarisation is similarly graded and increases with the

260 angular distance from the sun (Coemans et al. 1994). The integrated use of such cues is well

261 described among insects, including flies (Philipsborn et al. 1990; Weir and Dickinson 2012),

262 albeit typically in the context of navigation. Monarch butterflies, for example, rely on direct and

263 indirect skylight cues to calibrate their time-compensated sun compass during annual migrations

264 (Heinze and Reppert 2011; Reppert et al. 2016), while desert ants integrate solar intensity,

265 chromaticity, and polarisation during navigation to and from the nest (Müller and Wehner 2007;

266 Lebhardt and Ronacher 2015).

Though our results cannot directly test whether male L. cana make use of all available

268 cues when orienting, the suppression of their orientation bias with the obstruction of direct

269 sunlight — both artificial and natural—suggests that they first and foremost rely on the sun's

270 immediate position. This follows from the fact that gradients in each of polarization, and (to a

271 lesser extent) luminance and chromaticity are present even when the sun is not directly visible,

272 and many flies are able to use this information to guide behaviour in other contexts (Philipsborn

273 et al. 1990; Weir and Dickinson 2012). However, while there is reason to expect that males

274 should be able to orient their displays in non-optimal conditions, our modelling suggests that

275 there is no benefit in doing so. Signal luminance bears no relationship to solar orientation under

276 heavy cloud (Fig. 3d), so males should instead prioritise more consequential effects such as the

277 selection of suitable display backgrounds (White et al. 2020). Whether they do so remains to be

278 tested.

The importance of accurate solar orientation during courtship is heightened by L. cana's

280 use of structurally coloured ornaments. This is particularly true for wing interference patterns, 
281 the colour and luminance of which is a product of thin-film interference from the layering of

282 chitinous wing membranes (Shevtsova et al. 2011). Since these colours are highly specular (i.e.

283 dominated by mirror-like reflection) in nature, the absence of a point-source illuminant such

284 as the sun can render the patterns indistinguishable (Shevtsova et al. 2011). Males that are less

285 able to optimise their orientation would therefore suffer penalty not only in the quality of their

286 wing colouration (e.g. via reduced intensity), but the 'category' too, as the patterns will be

287 entirely absent at oblique illumination angles.

288 Since males' wings are also semi-transparent, their optimal appearance is equally

289 dependent on the background against which they are presented. Dark backgrounds—such as

290 seaweed common to the foreshore habitats of $L$. cana-are fit for purpose, as they minimise

291 transmitted light which would overwhelm the weakly reflective interference patterns. Recent

292 work has documented just such an effect with males preferentially displaying against darker

293 visual backgrounds, and the magnitude of signal/background contrast predicting mating success

294 (White et al. 2020). Though we did not track mating success here a comparable effect on fitness

295 is plausible, given that imprecision in either background selection or orientation relative to the

296 sun will produce a similarly diminished signal (Fig. 4c). It remains to be seen whether and to

297 what extent the sun's position and visual backgrounds are weighed and integrated during

298 courtship, but the accumulating evidence paints a striking picture of behavioural flexibility in

299 service of communication efficacy.

The behaviours dedicated to the presentation of iridescent faces and wings imply an

301 important role for the latter in sexual communication, though their precise function is as-yet

302 unresolved. As structurally coloured ornaments the macro-scale appearance of faces and WIP's

303 is closely tied to the nanoscale organisation of underlying structures (Kinoshita 2008). If males 
304 differentially vary in their ability to secure the conditions and/or material required to generate

305 such highly organised tissues, then the expression of facial and wing signals may be condition

306 dependent (Zahavi 1975; Keyser and Hill 1999; Shawkey et al. 2003).

308 bristles (unpublished data, see Frantsevich and Gorb 2006 for details in a sister species), the

309 brightness of which will largely depend on the size and density of scatterers (Giraldo and

310 Stavenga 2007; White et al. 2012; Stuart-Fox et al. 2018). The appearance of their wing

311 interference patterns is instead a product of coherent scattering, as noted above, with colour

312 differences across the wing surface chiefly determined by variation in the thickness of the

313 cuticular thin-films (Shevtsova et al. 2011). Both are constructed and fixed during ontogeny

314 using the pool of resources gathered during the larval stage, and so their final appearance may

315 reflect individual variation in foraging ability and condition more generally (e.g. Kemp 2008;

316 Kemp and Rutowski 2007). Recent meta-analytic evidence supports the potential for condition-

317 dependent expression among structural colours in general (White 2020), and also suggests that

318 the greater organisation demanded by coherent, as opposed to incoherent, scatterers makes them

319 particularly suitable as condition-dependent ornaments. This argues for WIPs as more probable

320 vectors of information on mate quality in L. cana than faces, with the latter potentially serving as

321 signals of sex or species identity. Despite convincing evidence for sexual selection in WIP

322 evolution (Katayama et al. 2014; Hawkes et al. 2019), however, direct tests of their function as

323 signals remain outstanding.

324 In addition to iridescent ornaments as conduits of information, a non-exclusive possibility

325 is that male behaviour is itself informative of mate quality. Courtship displays are likely to be

326 energetically costly as the cycle of pursuing, straddling, and displaying with a given female is 
327 often repeated several times before a clear outcome is achieved. Males clearly vary in their

328 ability achieve and sustain optimal orientations during courtship (Fig 2). If this variation is

329 dependent on male condition or 'quality' then their coloured ornaments may simply serve as

330 amplifiers of their behavioural performance. Displays against sub-optimal backgrounds or at

331 imprecise solar orientations would be readily apparent, particularly in the appearance of WIPs

332 (Shevtsova et al. 2011). Potential benefits to selective females are predictable since, as predators,

333 flight ability is central to prey capture in L. cana (Steidle et al. 1995; Pont 2019). This form of

334 condition-dependent variation in courtship effort, and corollary benefits to choosy viewers, is

335 well documented (Kotiaho 2000; Wagner and Hoback 1999; Jennions 1998). Similar effects are

336 known for variation in the precision of displays, such as in the quality of courts constructed by

337 bowerbirds (Endler et al. 2010; Kelley and Endler 2012), and the combination of limited-view

338 iridescence and precision displays in L. cana creates potential for a similar dynamic.

Our results extend a growing body of evidence which suggests extensive coevolution

340 between signals and display behaviours (Endler 1991; Endler and Thery 1996; Dakin and

341 Montgomerie 2009; Bortolotti et al. 2011; Sicsu et al. 2013; Klomp et al. 2017; White 2017;

342 Simpson and McGraw 2018). Notably, the strength of this link appears to vary with the extent of

343 fluidity in signalling environments and/or signals. That is, signals and environments which vary

344 dynamically across fine temporal or spatial scales are frequently coupled with flexible

345 behaviours to enhance the efficacy of information exchange. Iridescent signalling systems - such

346 as that examined here-fall at one extreme, with examples from insects (White et al. 2015), birds

347 (Endler and Thery 1996; Dakin and Montgomerie 2009; Simpson and McGraw 2018), and

348 guppies (Endler 1991) revealing considerable plasticity in display behaviour for optimising

349 signal delivery. Environments which vary unpredictably across fine spatial and temporal scales 
350 appear to favour similar adaptive solutions. The foreshores of $L$. cana offer a case in point, since

351 a single wave can add or remove tracts of seaweed which the flies use for shelter and signalling,

352 and thus entirely restructure their immediate visual environment.

353 Despite these few well-defined examples, the extent to which we can we can predict any

354 one of behaviour, habitat structure, or signal design from knowledge of the others is a long-

355 standing question (Poulton 1890; Lythgoe 1988; Endler and Thery 1996). One key to resolving

356 this challenge is a deeper appreciation of the spatio-temporal complexity of visual

357 communication. Exciting advances continue to be made in defining and integrating the spectral

358 (Maia et al. 2019; van den Berg et al. 2019), spatial (Caves et al. 2018; Stoddard and Osorio

359 2019), and temporal (Gruson et al. 2019a) features of signal production and perception, and

360 systems such as Lispe cana offer a promising context for empirical progress.

362 Acknowledgments

363 TEW is grateful to Elizabeth Mulvenna and Cormac White for their support.

\section{$365 \quad$ Funding}

366 None to report. 


\section{References}

Bortolotti, G. R., M. J. Stoffel, and I. Galvan. 2011. Wintering Snowy Owls Bubo scandiacus integrate plumage colour, behaviour and their environment to maximize efficacy of visual displays. Ibis 153:134-142.

Butterworth, N. J., P. G. Byrne, and J. F. Wallman. 2019. The blow fly waltz: Field and laboratory observations of novel and complex dipteran courtship behavior. Journal of Insect Behavior 1-11.

Butterworth, N. J., T. E. White, P. G. Byrne, and J. F. Wallman. 2020. Love at first flight: Wing interference patterns are species-specific and sexually dimorphic in blowflies (Diptera: Calliphoridae). bioRxiv.

Caves, E. M., N. C. Brandley, and S. Johnsen. 2018. Visual Acuity and the Evolution of Signals. Trends in Ecology \& Evolution 33:358-372.

Coemans, M., J. V. Hzn, and J. Nuboer. 1994. The relation between celestial colour gradients and the position of the sun, with regard to the sun compass. Vision research 34:1461-1470.

Dakin, R., and R. Montgomerie. 2009. Peacocks orient their courtship displays towards the sun. Behavioral Ecology and Sociobiology 63:825-834.

Endler, J. A. 1991. Variation in the appearance of guppy color patterns to guppies and their predators under different visual conditions. Vision Research 31:587-608.

Endler, J. A. 1992. Signals, signal conditions, and the direction of evolution. American Naturalist S125-S153.

Endler, J. A. 1993. Some general comments on the evolution and design of animal communication systems. Philosophical Transactions of the Royal Society of London Series B-Biological Sciences 340:215-225.

Endler, J. A., L. C. Endler, and N. R. Doerr. 2010. Great bowerbirds create theaters with forced perspective when seen by their audience. Current Biology 20:1679-1684.

Endler, J. A., and M. Thery. 1996. Interacting effects of lek placement, display behavior, ambient light, and color patterns in three neotropical forest-dwelling birds. American Naturalist 421-452.

Frantsevich, L., and S. Gorb. 2006. Courtship dances in the flies of the genus lispe (Diptera: Muscidae): From the fly's viewpoint. Archives of Insect Biochemistry and Physiology: Published in Collaboration with the Entomological Society of America 62:26-42. 
Giraldo, M. A., and D. G. Stavenga. 2007. Sexual dichroism and pigment localization in the wing scales of Pieris rapae butterflies. Proceedings of the Royal Society B: Biological Sciences 274:97-102.

Gruson, H., C. Andraud, W. Daney de Marcillac, S. Berthier, M. Elias, and D. Gomez. 2019a. Quantitative characterization of iridescent colours in biological studies: A novel method using optical theory. Interface Focus 9:20180049.

Gruson, H., T. White, and R. Maia. 2019b. Lightr: Import spectral data and metadata in R. Journal of Open Source Software 43:1857.

Hardie, R. C. 1986. The photoreceptor array of the dipteran retina. Trends in Neurosciences 9:419-423.

Hawkes, M., E. Duffy, R. Joag, A. Skeats, J. Radwan, N. Wedell, M. Sharma, et al. 2019. Sexual selection drives the evolution of male wing interference patterns. Proceedings of the Royal Society B 286:20182850.

Heinze, S., and S. M. Reppert. 2011. Sun compass integration of skylight cues in migratory monarch butterflies. Neuron 69:345-358.

Jennions, M. 1998. Variation in courtship rate in the fiddler crab Uca annulipes: Is it related to male attractiveness? Behavioral Ecology 9:605-611.

Johnsen, S. 2012. The optics of life: A biologist's guide to light in nature. Princeton University Press.

Katayama, N., J. K. Abbott, J. Kjærandsen, Y. Takahashi, and E. I. Svensson. 2014. Sexual

422 Kelley, L. A., and J. A. Endler. 2012. Illusions promote mating success in great bowerbirds. Science 335:335-338.

424 Kemp, D. J. 2008. Resource-mediated condition dependance in sexually dichromatic butterfly wing coloration. Evolution 62:2346-2358.

426 Kemp, D. J., and R. L. Rutowski. 2007. Condition dependence, quantitative genetics, and the potential signal content of iridescent ultraviolet butterfly coloration. Evolution 61:168-183.

Keyser, A. J., and G. E. Hill. 1999. Condition-dependent variation in the blue-ultraviolet coloration of a structurally based plumage ornament. Proceedings: Biological Sciences 
431 Kinoshita, S. 2008. Structural colors in the realm of nature. World Scientific Publishing 432 Company.

433 Klomp, D. A., D. Stuart-Fox, I. Das, and T. J. Ord. 2017. Gliding lizards use the position of the sun to enhance social display. Biology Letters 13:20160979.

435 Kotiaho, J. S. 2000. Testing the assumptions of conditional handicap theory: Costs and condition dependence of a sexually selected trait. Behavioral Ecology and Sociobiology 48:188-194.

Land, M. F. 1993. The visual control of courtship behaviour in the fly Poecilobothrus nobilitatus. Journal of Comparative Physiology A 173:595-603.

440 Lebhardt, F., and B. Ronacher. 2015. Transfer of directional information between the polarization compass and the sun compass in desert ants. Journal of Comparative Physiology A 201:599-608.

443 Lunau, K., A. Middelmann, and M. Pianka. 2006. Density and Food Resource Dependent Courtship Behaviour in the Fly Poecilobothrus nobilitatus L. (Diptera, Dolichopodidae) 10.

Lythgoe, J. N. 1988. Light and vision in the aquatic environment. In Sensory biology of aquatic animals (pp. 57-82). Springer.

447 Maia, R., H. Gruson, J. A. Endler, and T. E. White. 2019. Pavo 2: New tools for the spectral and spatial analysis of colour in R. Methods in Ecology and Evolution 10:1097-1107.

449 Marshall, S. A. 2012. Flies: The natural history \& diversity of Diptera. Firefly Books.

450 Mouchet, S. R., and P. Vukusic. 2018. Structural colours in lepidopteran scales. In Advances in insect physiology (Vol. 54, pp. 1-53). Elsevier.

452 Müller, M., and R. Wehner. 2007. Wind and sky as compass cues in desert ant navigation. Naturwissenschaften 94:589-594.

454 O’Neill, S. J., T. E. White, K. E. Lynch, and D. J. Kemp. 2019. Male guppies differ in daily frequency but not diel pattern of display under daily light changes. Behavioral Ecology and Sociobiology 73:157.

457 Philipsborn, A. von, T. Labhart, and others. 1990. A behavioural study of polarization vision in

458 the fly, Musca domestica. Journal of comparative physiology. A, Sensory, neural, and 459 behavioral physiology 167:737-743.

460 Pont, A. C. 2019. Studies on the australian muscidae (diptera). VIII. The genus lispe latreille, 461 1797. Zootaxa 4557:1-232.

462 Poulton, E. B. 1890. The colours of animals. Paul, Trench, Trübner \& Co, London. 
463 Prum, R. O. 2006. Anatomy, physics, and evolution of structural colors. Bird coloration 1:295353.

R Core Team. 2018. R: A language and environment for statistical computing. R Foundation for Statistical Computing, Vienna, Austria.

Reppert, S. M., P. A. Guerra, and C. Merlin. 2016. Neurobiology of monarch butterfly migration. Annual review of entomology 61.

Shawkey, M. D., A. M. Estes, L. M. Siefferman, and G. E. Hill. 2003. Nanostructure predicts intraspecific variation in ultraviolet-blue plumage colour. Proc R Soc B 270:1455-1460.

Shevtsova, E., C. Hansson, D. H. Janzen, and J. Kjærandsen. 2011. Stable structural color patterns displayed on transparent insect wings. Proceedings of the National Academy of Sciences 108:668-673. displays are associated with sunlight incidence. Behavioral Ecology and Sociobiology 67:1633-1642.

Simpson, R. K., and K. J. McGraw. 2018. It's not just what you have, but how you use it: Solar-positional and behavioural effects on hummingbird colour appearance during courtship. (G. Grether, ed.)Ecology Letters 21:1413-1422.

Steidle, J. L., K. Dettner, G. Hübner, A. Köpf, and J. Reinhard. 1995. The predaceous fly Lispe candicans (Diptera: Muscidae) and its chemically protected prey, the rove beetle Bledius furcatus (Coleoptera: Staphylinidae). Entomologia generalis 20:011-011. Quantitative Analysis. The American Naturalist 193:164-186.

Stuart-Fox, D., E. Newton, R. A. Mulder, L. D’Alba, M. D. Shawkey, and B. Igic. 2018. The microstructure of white feathers predicts their visible and near-infrared reflectance properties. PLoS ONE 13.

Thieurmel, B., and A. Elmarhraoui. 2019. Suncalc: Compute sun position, sunlight phases, moon position and lunar phase.

490 van den Berg, C. P., J. Troscianko, J. A. Endler, N. J. Marshall, and K. L. Cheney. 2019. Quantitative Colour Pattern Analysis (QCPA): A Comprehensive Framework for the

493 Vukusic, P. 2006. Structural colour in lepidoptera. Current Biology 16:R621-3. 
494 Wagner, W. E., and W. Hoback. 1999. Nutritional effects on male calling behaviour in the variable field cricket. Animal Behaviour 57:89-95.

496 Warrant, E. J. 2016. Matched filtering and the ecology of vision in insects. In The ecology of 497 animal senses (pp. 143-167). Springer.

498 Weaver, R. J., E. S. Santos, A. M. Tucker, A. E. Wilson, and G. E. Hill. 2018. Carotenoid metabolism strengthens the link between feather coloration and individual quality. Nature communications 9:73.

501 Weir, P. T., and M. H. Dickinson. 2012. Flying Drosophila orient to sky polarization. Current 502 biology 22:21-27.

503 White, T. E. 2017. Jewelled spiders manipulate colour-lure geometry to deceive prey. Biology $504 \quad$ letters 13:20170027.

505 White, T. E. 2020. Structural colours reflect individual quality: A meta-analysis. Biology $506 \quad$ Letters.

507 White, T. E., J. Macedonia, D. Birch, J. Dawes, and D. J. Kemp. 2012. The nanoanatomical

508 basis of sexual dimorphism in iridescent butterfly colouration. Australian journal of 509 zoology 60:101-107.

510 White, T. E., N. Vogel-Ghibely, and N. J. Butterworth. 2020. Flies exploit predictable 511 perspectives and backgrounds to enhance iridescent signal salience and mating success. 512 The American Naturalist 0:0.

513 White, T. E., J. Zeil, and D. J. Kemp. 2015. Signal design and courtship presentation coincide 514 for highly biased delivery of an iridescent butterfly mating signal. Evolution 69:14-25.

515 Zahavi, A. 1975. Mate selectiona selection for a handicap. Journal of theoretical Biology $516 \quad 53: 205-214$.

517 Zeil, J. 1983. Sexual dimorphism in the visual system of flies: The compound eyes and neural 518 superposition in Bibionidae (Diptera). Journal of Comparative Physiology A: 519 Neuroethology, Sensory, Neural, and Behavioral Physiology 150:379-393.

520 Zimmer, M., O. Diestelhorst, and K. Lunau. 2003. Courtship in long-legged flies (Diptera: 521 Dolichopodidae): Function and evolution of signals. Behavioral Ecology 14:526-530. 


\section{Figures and Tables}
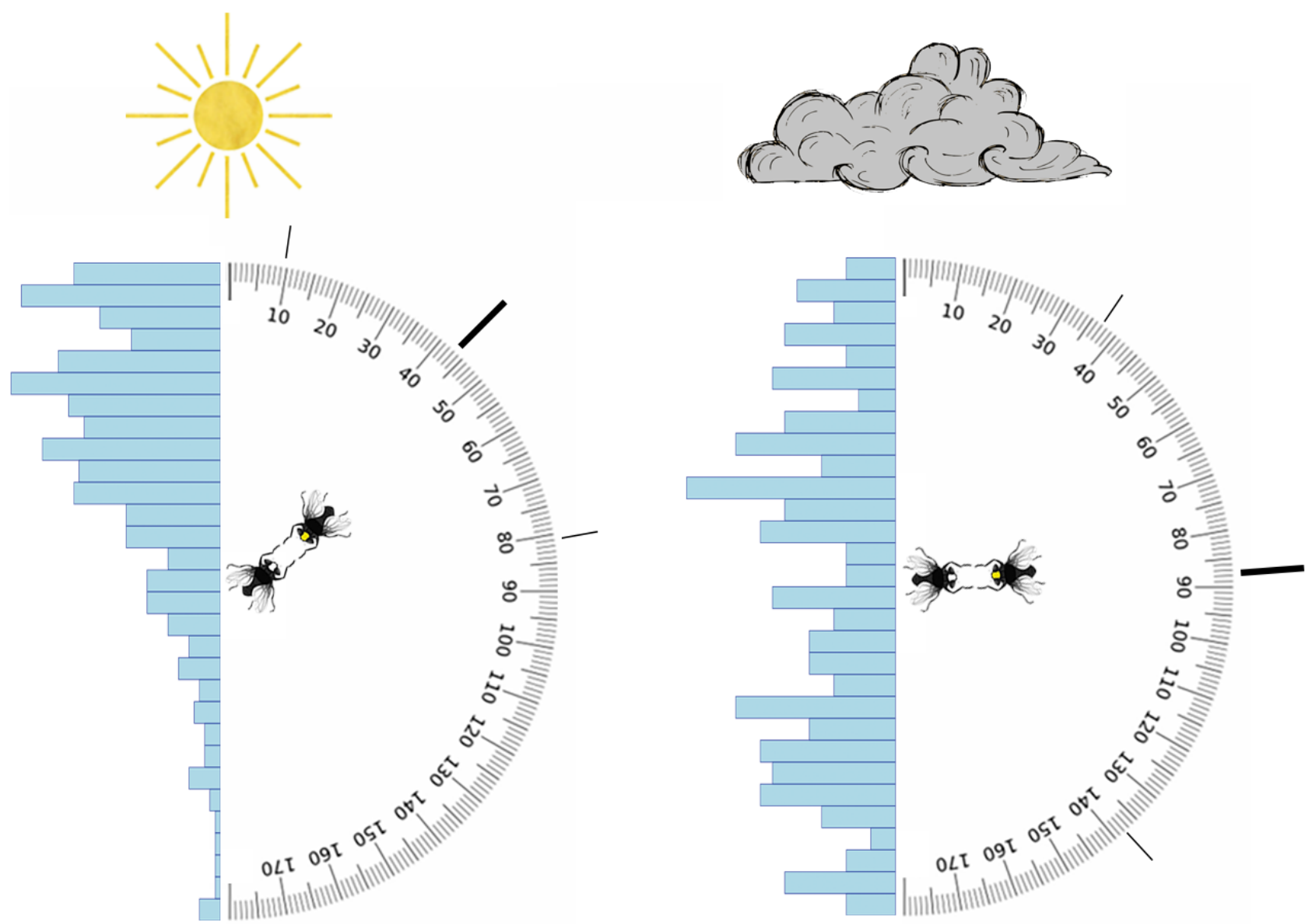

533 Figure 1: The orientations of Lispe cana during courtship displays under full sun (left) and

534 heavy cloud (right). When displaying, male flies (white face) position themselves immediately in

535 front of females (yellow face), and twist their wings forward in a seemingly ritualized 'dance'.

536 Angles denote the offset of the male midline from the solar azimuth during courtship, with $0^{\circ}$

537 representing a display directly toward the sun, and $180^{\circ}$ directly away. Histograms show the

538 distribution of male offsets from the sun under both conditions ( $n=450$ full sun, $n=227$ heavy

539 cloud), with the median \pm standard deviation indicated by solid lines. 


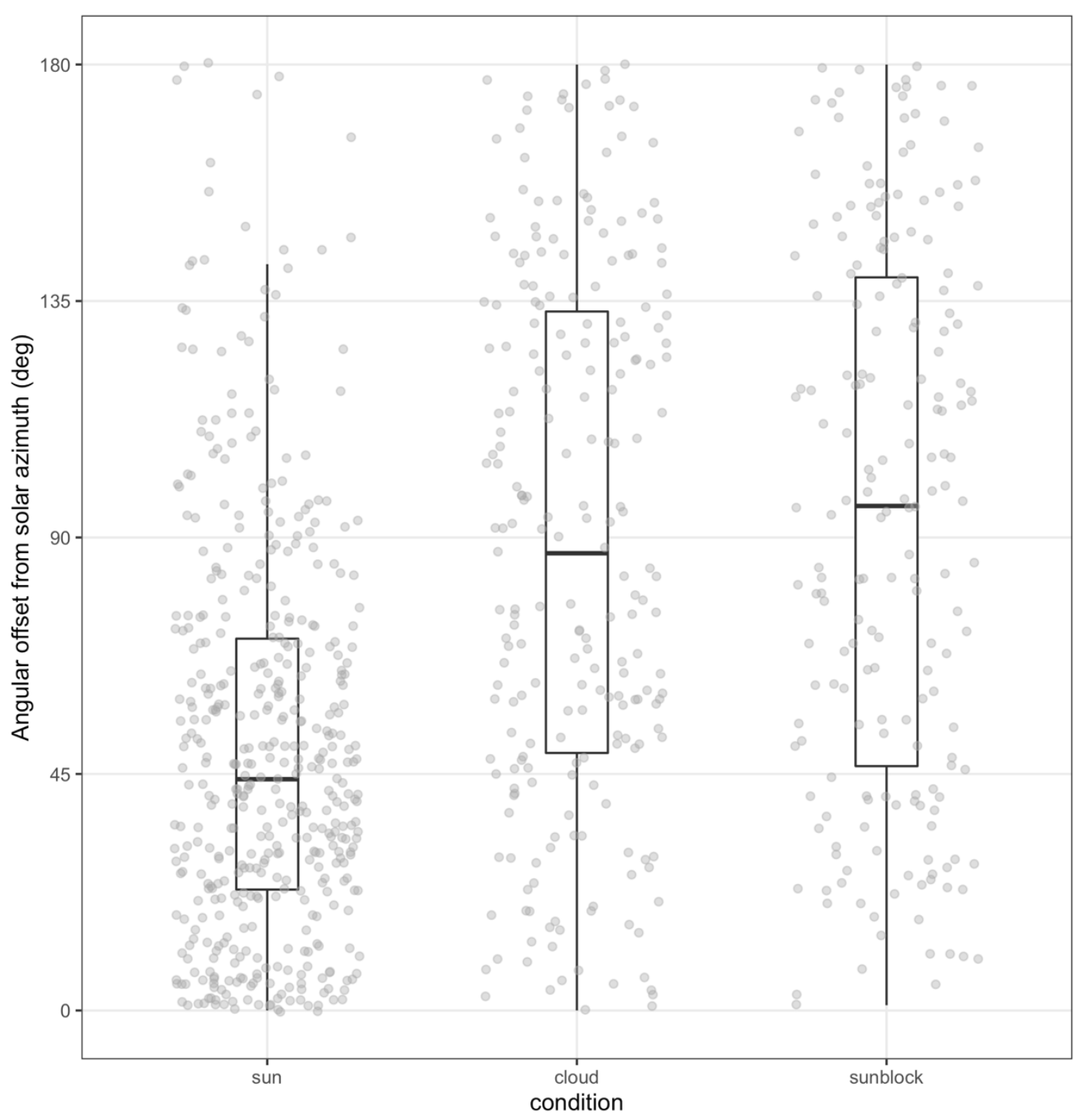

555 Figure 2: The orientation of displaying male Lispe cana with respect to the sun in conditions of

556 full sun, heavy cloud, or experimentally obscured sunlight. Orientations are expressed as offsets

557 from the solar azimuth, pooled across the 0730 - 1630 observation period, with $0^{\circ}$ representing

558 males presenting their iridescent signals directly toward the sun and $180^{\circ}$ directly away. 


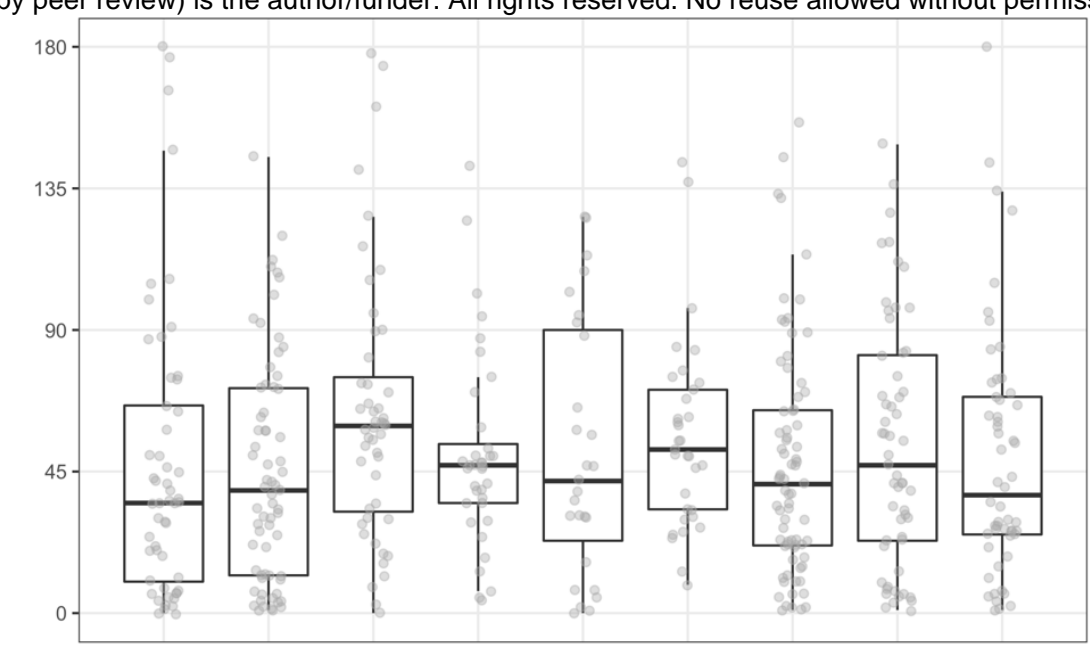

\section{6}

567

568

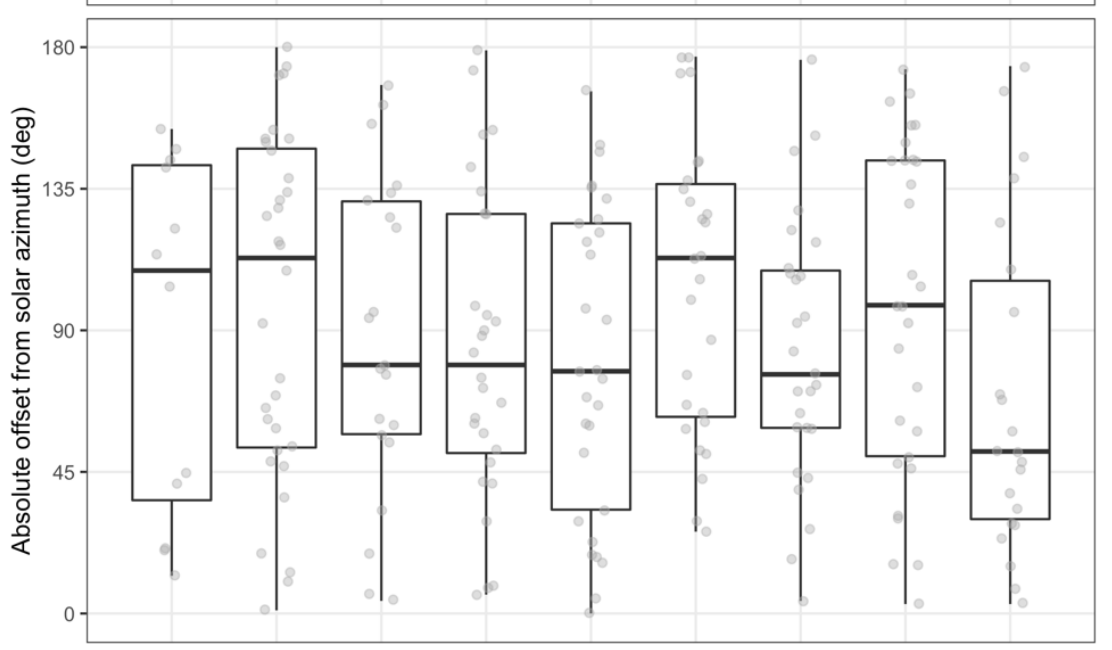

571

569

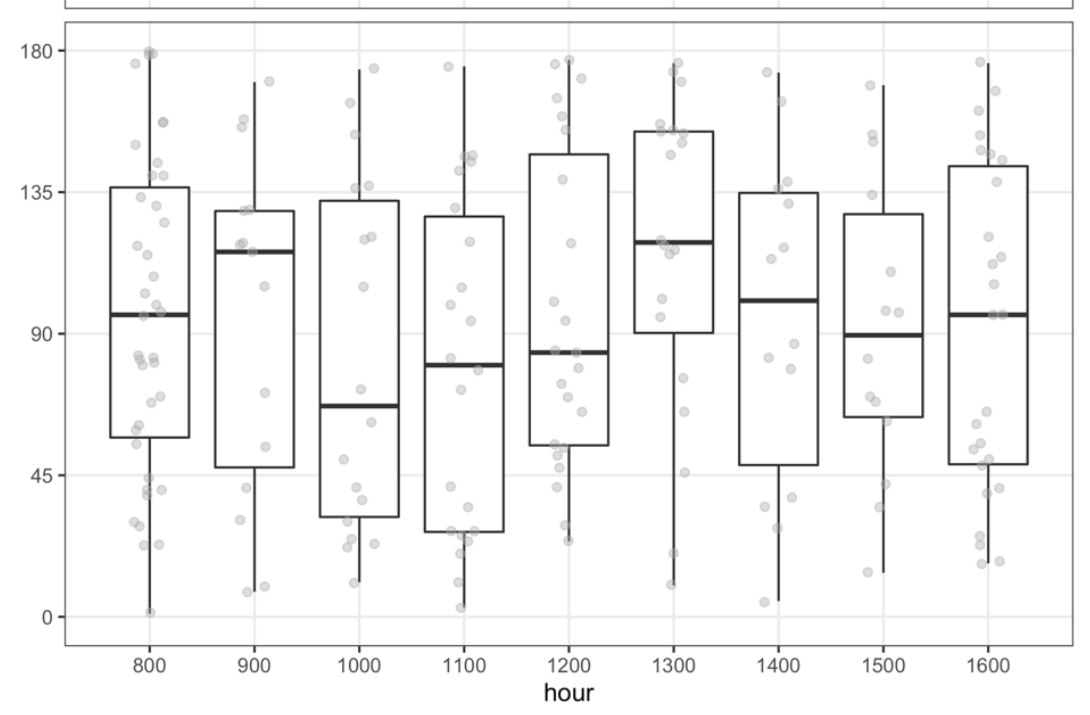

578 Figure 3: The orientation of displaying male Lispe cana in conditions of full sun (top), heavy

579 cloud (middle), or experimentally obscured sunlight (bottom), pooled into hourly periods.

580 Orientations are expressed as offsets from the solar azimuth, with $0^{\circ}$ representing males

581 presenting their iridescent signals directly toward the sun and $180^{\circ}$ directly away. 

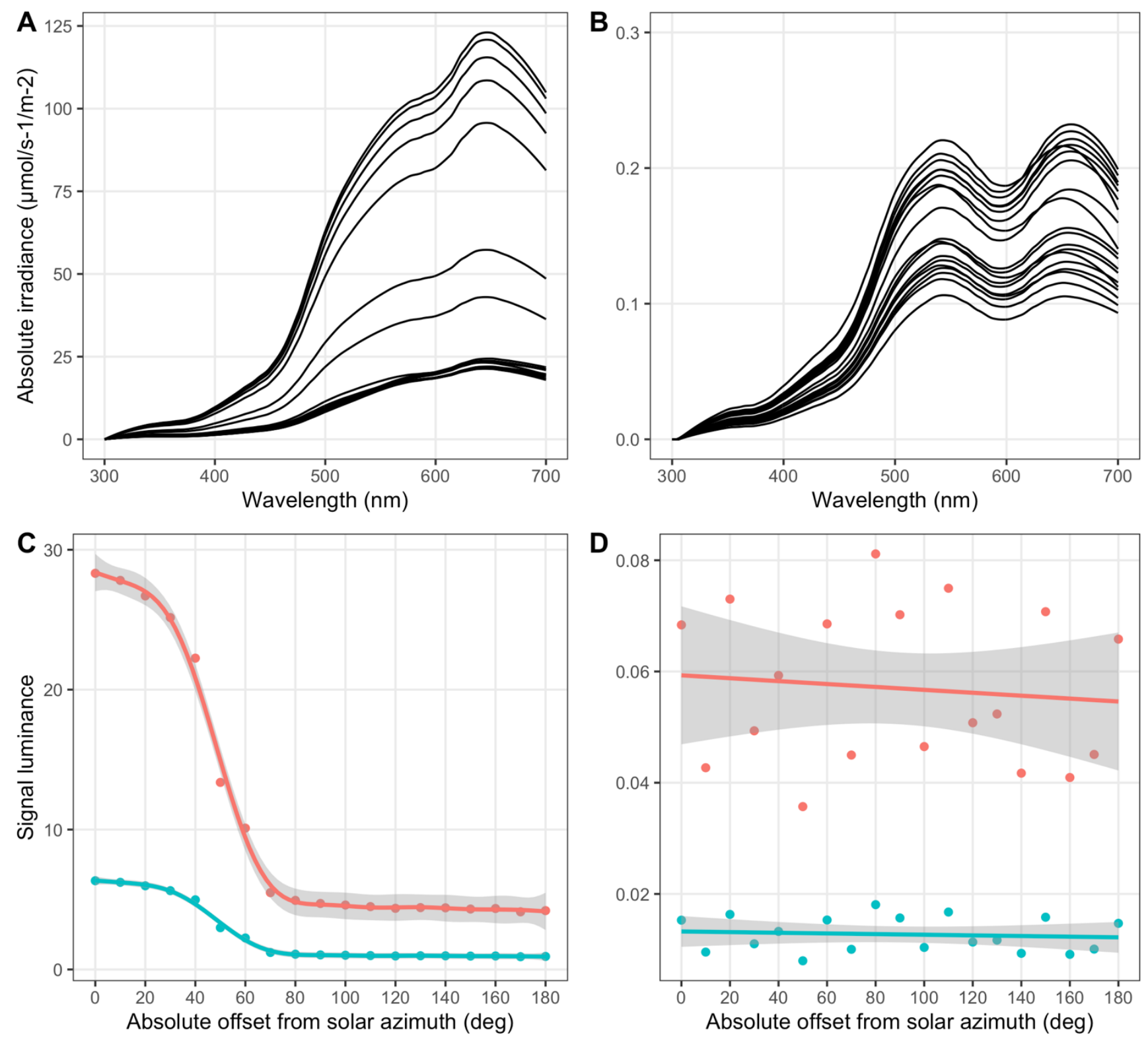

583 Figure 4: Solar irradiance and male signal luminance as a function of solar orientation. Solar 584 irradiance in conditions of (a) full sun and (b) heavy cloud, recorded at $0^{\circ}-180^{\circ}$ offset from the 585 solar azimuth in $10^{\circ}$ increments. Below is the luminance of male faces (red) and wings (green) 586 across the same angular range, as modelled under full sun (c) or cloud (d). Lines denote 587 generalised additive model fits \pm standard errors. Not differing y axes across all panels. 
589 Table 1: Parameter estimates and summary statistics from a generalised linear mixed

590 model examining the predictors of male display orientation in Lispe cana. Included are the

591 experimental condition (full sun, heavy cloud, and artificially obscured sunlight) and solar

592 elevation. Julian date was included as a random effect with a variance of 0.301 . Full model

593 conditional $\mathrm{R}^{2}=0.169$.

\begin{tabular}{lccccc}
\hline Fixed effects & Estimate & SE & df & $\boldsymbol{t}$ & $\boldsymbol{p}$ \\
\hline intercept & 8.389 & 0.771 & 853 & 10.88 & $<0.001$ \\
condition (sun) & -3.115 & 1.336 & 9 & -2.743 & 0.023 \\
condition (sunblock) & 0.425 & 1.014 & 9 & 0.419 & 0.685 \\
solar elevation & 0.011 & 0.016 & 853 & 0.712 & 0.476 \\
condition (sun) x solar elevation & 0.014 & 0.023 & 853 & 0.624 & 0.533 \\
condition (sunblock) x solar elevation & -0.003 & 0.020 & 853 & -0.191 & 0.849 \\
\hline
\end{tabular}

594 